\title{
HDR Video Quality Evaluation of HEVC and VP9 Codecs
}

\author{
Glenn Herrou ${ }^{\star}$, Wassim Hamidouche ${ }^{\dagger}$, Xavier Ducloux ${ }^{\star}$ \\ ${ }^{\star} \mathrm{b}<>$ com, Cesson-Sevigne, France \\ ${ }^{\dagger}$ IETR / INSA de Rennes, Rennes, France \\ \{glenn.herrou, xavier.ducloux $\} @$ b-com.com, wassim.hamidouche@insa-rennes.fr
}

\begin{abstract}
Current increasing effort in the television industry towards High Dynamic Range (HDR) imaging has raised the issue of the compression of HDR content. Offering a higher peak luminance and wider color gamut, HDR video introduces new challenges to the state-of-the-art video codecs such as High Efficiency Video Coding (HEVC) or VP9, which have been designed and optimized for the compression of Standard Dynamic Range (SDR) content. This study presents a performance comparison between HEVC and VP9 in the HDR context through both objective and subjective evaluations. The experimental objective results have shown that $\mathrm{HEVC}$ offers from $0.6 \%$ to $38.2 \%$ bit rate savings over VP9 depending on the objective metric which is used. The subjective study demonstrated that, on average, bit rate savings greater than $47.7 \%$ can be achieved by HEVC for the same perceived quality as VP9.
\end{abstract}

Index Terms-HDR, video compression, HEVC, VP9, subjective evaluation, video quality assessment

\section{INTRODUCTION}

Over the last decade, television industry has been pushing towards High Dynamic Range (HDR) imaging and display systems. The motivation behind this new trend is the delivery of content close to what would be perceived by the human eye, which is sensible to luminance levels from $10^{-6}$ to $10^{8} \mathrm{~cd} / \mathrm{m}^{2}$ [1]. However, in a single scene, the instantaneous sensitivity of the Human Visual System (HVS) can reach a contrast of 10000:1, far beyond the dynamic range allowed by the majority of existing capture and display devices known as Standard Dynamic Range (SDR) - in the ranges 100:1 and 1000:1. A new generation of devices, supporting HDR, recently emerged and raised the need of defining new tools for efficient delivery of HDR content. HDR compression has thus become a topic of interest for the video coding community.

State-of-the-art video codecs such as High Efficiency Video Coding (HEVC) [2] and VP9 [3] have been traditionally designed and evaluated for SDR (8 bits and low peak brightness) content like their predecessors Advanced Video Coding (AVC) [4] and VP8 [5], respectively. However, HEVC and VP9 codecs enable the processing of 10-bit per component signal through the Main $10 \mathrm{HEVC}$ profile and the profile 2 of VP9, which is the minimum bit depth recommended to encode HDR signals with a low visible quantization distortion [6], [7]. Therefore, HEVC and VP9 can easily be inserted in a HDR coding scheme, along with pre and post processing operations including a Transfer Function (TF) such as ST.2084 [8], standardized by the Society of Motion Picture and Television
Engineers (SMPTE), or the STD-B67 [9], standardized by the Association of Radio Industries and Businesses (ARIB), necessary for the transmission and compression of HDR signals.

The performances of HEVC and VP9 have been compared in [10], [11] for SDR content, showing a higher coding efficiency for the Motion Picture Experts Group (MPEG) codec HEVC. Furthermore, Azimi et al. [12] evaluated the performance of HEVC in the HDR context, comparing the HDR coding chain defined in the MPEG Call for Evidence (CfE) on HDR [13], which is also used in this paper, and a backward compatible scheme where the HDR content is tone mapped to a SDR signal before the encoding process. However, little is known on the performance efficiency of VP9 in a HDR context compared to HEVC, especially since the definition of additional coding tools dedicated to HDR content coding in HEVC [14].

In this paper, we study and compare the performance of the latest implementations of both HEVC and VP9 for HDR encoding, providing both objective and subjective evaluations over a wide range of High Definition (HD) (1920x1080) and Ultra High Definition (UHD) (3840x2160) HDR video sequences. Ultimately, this study showed that, for the compression of HDR content, HEVC offers a substantially better coding efficiency in average relative to VP9, in terms of perceived visual quality at the same bit rate.

The rest of this paper is organized as follows. Section II provides a brief background on state-of-the-art video codecs as well as on HDR content processing and its specific coding tools. Section III describes the details of our experiment. Section IV presents and discusses the results and Section V concludes this paper.

\section{BACKGROUND AND RELATED WORK}

This section provides a brief background on state-of-the-art video coding technologies and HDR compression scheme as well as an overview of the coding tools specifically designed for HDR content.

\section{A. Video Coding Standards}

The first version of the HEVC standard [2] was finalized in January 2013 by ITU-T Video Coding Experts Group (VCEG) and ISO/IEC MPEG under a partnership known as Joint Collaborative Team on Video Coding (JCT-VC). It enables 


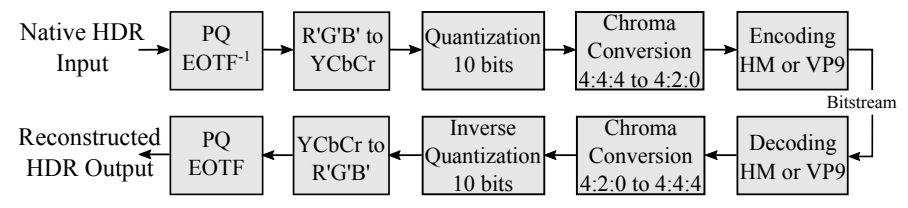

Fig. 1. End-to-end HDR coding chain.

up to a $50 \%$ gain in terms of subjective video quality over AVC [15], [16]. The second version of the standard brought support of high bit depth encoding (up to 14 bits) and other chroma subsampling formats $(4: 2: 2$ and 4:4:4). These new features, together with the consequent bit rate gains provided by HEVC, allows for the deployment of new services such as broadcasting of UHD programs.

In parallel, Google ${ }^{\circledR}$ Inc. has developed its own next generation video codec called VP9, which is open source, royaltyfree and designed for web applications. The first profile of VP9 was finalized in June 2013, aiming for a $50 \%$ bit rate reduction for the same visual quality compared to its predecessor VP8. Several profiles have been added in the months following the release of the codec, enabling high bit depth coding and support of additional chroma subsampling formats .

\section{B. High Dynamic Range Processing Chain}

An end-to-end coding and decoding chain for HDR video, depicted in Figure 1, has been defined in the MPEG CfE for HDR Video Coding [13]. This processing chain takes as input and output a RGB linear-light signal, typically OpenEXR format [17] (half precision floating point). Before feeding the source signal to the encoder, several pre-processing steps are performed. First, the input is mapped using the considered TF. In this study, only the ST.2084 transfer function has been considered since STD-B67 was not supported by the monitor used for the evaluation at the time of our experiment. The nonlinear R' $\mathrm{G}^{\prime} \mathrm{B}$ ' signal is converted to $4: 4: 4 \mathrm{Y}^{\prime} \mathrm{CbCr}$, quantized to 10-bit representation and finally subsampled to 4:2:0.

Then, the 4:2:0 10-bit $\mathrm{Y}^{\prime} \mathrm{CbCr}$ signal can be encoded and decoded using the considered codec and finally converted back to RGB linear-light before displaying the content. All pre and post processing steps can be performed using the HDRTools suite [18].

\section{HDR Video Coding Optimization}

In this section we describe two non-normative coding features integrated in HEVC in order to improve the coding efficiency for HDR video content.

1) Chroma QP Offset: Due to the inherent lower magnitude of chroma components $(\mathrm{Cb}$ and $\mathrm{Cr})$ compared to the luma component, lower Quantization Parameter (QP) values for $\mathrm{Cb}$ and $\mathrm{Cr}$ are needed to avoid the complete loss of chroma information when high QPs are used. In [19], it was shown that the use of chroma QP offsets could improve the visual quality when encoding HDR content. A study on the QP offset values was presented in [20], showing that the optimal QP offsets depend on the difference between the capture
TABLE I

TEST SEQUENCES AND THEIR CHARACTERISTICS

\begin{tabular}{lcccc}
\hline Sequence & $\begin{array}{c}\text { Resolution } \\
\text { (width } \\
\text { height })\end{array}$ & $\begin{array}{c}\text { Frame } \\
\text { rate } \\
(\mathrm{fps})\end{array}$ & Frames & $\begin{array}{c}\text { Peak } \\
\text { Luminance } \\
\left(\mathrm{cd}^{2} \mathrm{~m}^{2}\right)\end{array}$ \\
\hline Boat1 & $3840 \times 2160$ & 50 & 500 & \\
Pontoon & $3840 \times 2160$ & 50 & 500 & 1000 \\
CatRobot1 & $3840 \times 2160$ & 60 & 600 & \\
Drums2 & $3840 \times 2160$ & 50 & 500 & 1000 \\
\hline Market3 & $1920 \times 1080$ & 50 & 400 & \\
BalloonFestival & $1920 \times 1080$ & 24 & 240 & 4000 \\
Rowing2 & $1920 \times 1080$ & 60 & 600 & \\
FreeSardines1 & $1920 \times 1080$ & 60 & 600 & \\
\hline Market3 & $1920 \times 1080$ & 50 & 400 & \\
Sunrise & $1920 \times 1080$ & 25 & 200 & 200 \\
FireEater2 & $1920 \times 1080$ & 25 & 240 & \\
Tibul2 & $1920 \times 1080$ & 30 & & \\
\hline
\end{tabular}

and representation color spaces, as well as on the luma QP magnitude. Thus, a formula was proposed to compute the QP offsets for chrominance components:

$$
\begin{aligned}
& \Delta Q P_{C b}=\max \left(-12, \operatorname{round}\left(c_{C b} *(k * Q P+l)\right)\right) \\
& \Delta Q P_{C r}=\max \left(-12, \operatorname{round}\left(c_{C r} *(k * Q P+l)\right)\right)
\end{aligned}
$$

where $k=-0.46, l=9.26, c_{C b}=1.14$ and $c_{C r}=1.78$ when BT.709 color space in BT.2020 container is considered.

2) Average-luma Controlled Adaptive QP: HEVC, like most existing codecs, has been designed for encoding SDR content using BT.709 color space whereas the recommended container for HDR content is BT.2020 since it offers wider color gamut. A recent study in [21] showed that the quantization noise introduced by the coding algorithm is not linear between BT.709 and BT.2020 color spaces. Specifically, it was pointed out that adapting the QP based on the average luma value, at a macroblock level, could significantly improve the quantization step in terms of quality loss when using the BT.2020 color space, nearly achieving the performance obtained using the well controlled BT.709 color space.

\section{Methodology And Test Settings}

This section provides an overview of the test material used, the selected encoder configurations, the considered objective metrics as well as the subjective test settings and logistics.

\section{A. Selection of Test Sequences}

In order to evaluate the performance of HEVC and VP9 video codecs in an HDR context, twelve sequences have been selected. The details of the test sequences are provided in Table I. Four UHD sequences $(3840 \times 2160)$ and four HD sequences (1920x1080) are used for both the objective and subjective tests. The remaining four sequences (HD 4000 $\mathrm{cd} / \mathrm{m}^{2}$ ) have been selected to expand the scope of the study to sequences with higher peak luminance. These $4000 \mathrm{~cd} / \mathrm{m}^{2}$ peak luminance sequences were not included in the subjective test due to the characteristics of the available display (limited to $1000 \mathrm{~cd} / \mathrm{m}^{2}$ ). 
TABLE II

SETTINGS FOR HEVC REFERENCE SOFTWARE ENCODER.

\begin{tabular}{lc}
\hline Coding Options & Parameter \\
\hline Encoder Version & HM 16.7 CE1-anchor v3.2 \\
Profile & Main 10 \\
R/D Optimization & Enabled \\
GOP & 8 \\
Hierarchical Encoding & Enabled \\
Temporal Layers & 4 \\
Intra Period & $1 \mathrm{sec}$ \\
Coding Unit Size/Depth & $64 / 4$ \\
Transform Unit Size (Min/Max) & $4 / 32$ \\
Deblocking Filter & Enabled \\
Sample Adaptive Offset (SAO) & Enabled \\
Rate Control & Disabled \\
\hline
\end{tabular}

The sequences have been selected from various sources and have different spatio-temporal characteristics, leading to different compression algorithm behaviours. The database offers a wide range of natural content. Four HDR sequences in the content pool - CatRobot1, Drums2, Rowing2 and FreeSardines 1 - have been obtained after a tone expansion of the SDR version using the algorithm proposed in [22].

\section{B. Encoder Configurations}

The test sequences have been compressed using the HEVC reference software (HM16.7) [23] with the improvements proposed by MPEG for HDR content through the Core Experiment 1 (CE1) anchor, and the VP9 software (v1.5.0) [5] provided by the WebM Project. Encodings have been performed using 4:2:0 subsampling and 10-bit per component per sample in the Y' $\mathrm{CbCr}$ color space (Main $10 \mathrm{HEVC}$ profile and VP9 profile 2), according to the coding chain described in Figure 1. Random access configuration, with an intra period of approximately one second and a Group Of Pictures (GOP) size of eight, has been used for both codecs. Backward prediction was enabled by allowing hierarchical GOP structures with four temporal layers in $\mathrm{HEVC}$ and by turning on alternate reference frames in VP9. The detailed encoder configurations are summarized in Tables II and III. The reader is referred to [24] to obtain more information on all VP9 commands presented in Table III.

Fixed QP configuration has been chosen for both objectives and subjective tests. For each sequence, four different QP values were selected so that the bit rates of both codecs were similar for each test point.

\section{Objective Study}

Since there is currently no agreement on which metric should be used to evaluate HDR content, several metrics available in the HDRTools software package [18] have been used in this study: tPSNR, mPSNR, DeltaE, HDR Video Quality Measure (HDR-VQM) [25] and Visual Information Fidelity (VIF) [26].

The objective tests have been carried out on the twelve sequences described in Table I, using four different QPs per sequence, for both HEVC and VP9 codecs, representing a
TABLE III

SETTINGS FOR VP9 REFERENCE SOFTWARE ENCODER.

\begin{tabular}{|c|c|}
\hline Version & VP9 Encoder v1.5.0-520-g6261fcf, November 2015 \\
\hline \multirow{8}{*}{ Parameters } & -- cpu-used $=0$ \\
\hline & -- codec $=$ vp9 $\quad--$ profile $=2 \quad--$ passes $=2$ \\
\hline & - -height $=\$$ height \\
\hline & $--\mathrm{fps}=\$$ frameRate $/ 1$ \\
\hline & -- auto-alt-ref $=1$ \\
\hline & -- arnr-maxframes $=7$ \\
\hline & --input-bit-depth $=10$ \\
\hline & $--\mathrm{kf}-\min -\mathrm{dis} t=\$$ intraPeriod $--\mathrm{kf}-\mathrm{max}-\mathrm{dist}=\$$ intraPeriod \\
\hline
\end{tabular}

wide range of quality. The same parameters (three lower QPs of each sequence) have been used in the subjective study. Bjøntegaard Delta (BD) rates [27] have been computed for each metric to evaluate the coding algorithms.

\section{Subjective Test Methodology}

In this study, the purpose of the subjective tests is to evaluate the quality of the two video codecs under consideration over a wide range of visual quality. Thus, according to ITUT Recommendation P.910 [28], the recommended evaluation method is Degradation Category Rating (DCR), which is commonly used for this type of context [15], [10]. The quality rating scale used is made of 11 levels [28], ranging from 0 (lowest quality) to 10 (highest quality), as described in [15].

Subjective tests for different categories of video sequences were carried out in separate test sessions. Each session of the DCR method consisted of consecutive Basic Test Cells (BTCs) displayed on the monitor. A BTC is composed of four presentations of the sequence under test. The original video clip and coded version are played twice, alternatively, with the original sequence first. A two-second message is displayed before each occurrence of the clip, announcing to the viewer which sequence (A or B) will be played next. After the four presentations, a message asking the viewers to vote is displayed for five seconds. The structure of a BTC is depicted in Figure 2.

Each session was composed of 28 BTCs. The first two BTCs were used as coherence check, showing original versus original content. The next two BTCs represented the stabilization phase and were selected to show the range of quality seen during the test. The results from these BTCs were not included in the analysis. BTCs were randomly ordered to avoid the repetition of the same content and to spread the quality as much as possible throughout the whole test.

The tests were conducted in a controlled laboratory environment, following the the ITU-R Recommendation BT.50013 [29]. The HDR display used is the OLED 32-inch professional monitor Sony BVM-X300. To feed the video content to the monitor, a high performance video player capable of displaying uncompressed UHD content at high frame rates was used. A total of 33 participants - 16 for the HD session and 17 for the UHD session - took part in the study, aged from 23 to 55 years old, with (corrected to) normal visual 


\begin{tabular}{|c|c|c|c|c|}
\hline Original & Coded & Original & Coded & Vote - N \\
\hline 8 -10 seconds & 8 -10 seconds & $8-10$ seconds & 8 -10 seconds & 5 seconds \\
\hline
\end{tabular}

Fig. 2. Basic Test Cell for subjective evaluation

acuity and normal color vision. The participants were placed at a distance of 3 times the screen height.

\section{Results}

The quality of decoded HDR sequences with respect to the original content has been evaluated objectively with various metrics. The metric-based BD-Rates have been computed for each sequence, as shown in Table IV. A negative value denotes bit rate savings for HEVC over VP9 while a positive value indicates a bit rate overhead for HEVC. Overall, every metric shows a compression gain of HEVC with respect to VP9 despite large differences in average BD-Rates values depending on the metric considered. In particular, HDR-VQM values vary significantly from one test sequence to another, sometime yielding in a better quality for VP9 codec while the results from the other metrics indicate a better efficiency of HEVC for all sequences. Furthermore, it can be observed that the compression gain of HEVC over VP9 is in average greater by 2 to $15 \%$ for UHD content compared to HD sequences. This trend would need to be confirmed using UHD and HD versions of the same content. In addition, the BD-Rate gains of HEVC tend to decrease in average for the compression of $4000 \mathrm{~cd} / \mathrm{m}^{2}$ peak luminance content.

The results of the subjective tests are evaluated in terms of Mean Opinion Score (MOS), obtained by averaging scores of all subjects for a given test point, with the associated $95 \%$ confidence interval. Two outliers were detected in the UHD session and their results were discarded from further analysis.

Figure 3 shows the results of the subjective tests in terms of MOS values versus the bit rate used to encode the HEVC or VP9 HDR bitstreams. It can be observed that HEVC obtains significantly higher MOS values than VP9 for most test sequences. The only sequence where VP9 achieves a similar observed quality for roughly the same bit rate is Boat1. For this sequence, subjective scores are above 9.5, which corresponds to a visually nearly lossless compression, for all test points except the lowest bit rate using VP9. This bit rate (approximately $3 \mathrm{Mbps}$ ) is quite low for the compression of UHD content and results in visible blocking artifacts for VP9 codec. However, HEVC keeps a good quality at this low bit rate, with no apparent distortion.

In general, at high bit rates, HEVC performs really well on every type of video content whereas temporal instability, i.e. visible activity at the beginning of each intra period, can be observed on content with large static areas (sky, rendered wall, etc.) using VP9 codec at every test point. This temporal instability is not present in the HEVC coded sequences thanks to the HDR-specific tool Average-luma controlled adaptive $Q P$ available in the CE1 anchor v3.2. At lower bit rates, traditional blocking artifacts and blurred areas appear with both HEVC
TABLE IV

BD-RATES FOR OBJECTIVE EVALUATION RESULTS

\begin{tabular}{lccccc}
\hline \multirow{2}{*}{ Sequence } & \multicolumn{5}{c}{ BD-Rates for HEVC over VP9 } \\
& tPSNR- & mPSNR & DE100 & VQM & VIF \\
& Y & & & & \\
\hline Boat1 & $-29.8 \%$ & $-35.3 \%$ & $-27.6 \%$ & $-27.2 \%$ & $-40.3 \%$ \\
Pontoon & $-27.8 \%$ & $-37.9 \%$ & $-38.3 \%$ & $-1.3 \%$ & $-33.6 \%$ \\
CatRobot1 & $-17.4 \%$ & $-21.0 \%$ & $-47.8 \%$ & $-12.2 \%$ & $-28.3 \%$ \\
Drums2 & $-15.2 \%$ & $-19.0 \%$ & $-52.8 \%$ & $-12.0 \%$ & $-22.2 \%$ \\
\hline Market3 & $-18.3 \%$ & $-14.0 \%$ & $-56.5 \%$ & $4.1 \%$ & $-31.7 \%$ \\
BalloonFestival & $-26.9 \%$ & $-21.4 \%$ & $-12.1 \%$ & $-5.0 \%$ & $-36.2 \%$ \\
Rowing2 & $-22.1 \%$ & $-26.1 \%$ & $-62.5 \%$ & $-22.2 \%$ & $-34.4 \%$ \\
FreeSardines1 & $-4.6 \%$ & $-9.8 \%$ & $-40.6 \%$ & $33.3 \%$ & $-11.4 \%$ \\
\hline Market3 & $-16.8 \%$ & $-16.2 \%$ & $-58.8 \%$ & $4.5 \%$ & $-30.3 \%$ \\
Sunrise & $-8.6 \%$ & $8.3 \%$ & $-28.0 \%$ & $13.7 \%$ & $-41.1 \%$ \\
FireEater2 & $-7.8 \%$ & $16.4 \%$ & $-7.4 \%$ & $20.4 \%$ & $-10.2 \%$ \\
Tibul2 & $-17.2 \%$ & $-14.6 \%$ & $-26.4 \%$ & $-3.5 \%$ & $-26.0 \%$ \\
\hline Average UHD & $-22.5 \%$ & $-28.3 \%$ & $-41.1 \%$ & $-13.2 \%$ & $-31.1 \%$ \\
Average HD & $-17.9 \%$ & $-17.8 \%$ & $-42.9 \%$ & $2.6 \%$ & $-28.4 \%$ \\
Average 4000 nits & $-12.6 \%$ & $-1.5 \%$ & $-30.2 \%$ & $8.8 \%$ & $-26.9 \%$ \\
\hline Overall & $-17.7 \%$ & $-15.9 \%$ & $-38.2 \%$ & $-0.6 \%$ & $-28.8 \%$ \\
\hline & & & & & \\
\hline
\end{tabular}

and VP9 codecs resulting in a decrease of MOS values for almost every sequence. Chroma artifacts, which are amplified by the HDR representation format, are also visible on bright areas. These color artifacts appear with both codecs, especially at low bit rates, but are much less noticeable with HEVC thanks to the HDR-specific tool Chroma Qp Offset available in the chosen reference software implementation. However, this type of distortion is generally less annoying than other artifacts and thus often not detected by non-expert viewers.

Overall, BD-Rate measures for MOS values show that bit rate savings, for HEVC relative to VP9, greater than $45.4 \%$ and $50.1 \%$ can be achieved for the UHD and HD content, respectively. Though, the average BD-Rate gain for the UHD sequences is not fully reliable since only a minimum average BD-Rate value can be obtained. Indeed, proper BD-Rate values cannot be computed for every sequence due to the lack of overlapping MOS ranges in some cases.

\section{CONClusion}

In this paper, a detailed description of the objective and subjective evaluations conducted to assess the performance of two video codecs, HEVC, with its latest HDR-specific coding tools, and VP9, for high dynamic range video coding has been presented. Experimental results have shown that HEVC performs better than VP9 in terms of both objective measures and subjective scores. The objective evaluation demonstrated a bit rate reduction of $0.6 \%$ to $38.2 \%$ for HEVC relative to VP9, for the same measured quality. According to the subjective evaluation, HEVC achieved better performance with average bit rate savings greater than $47.7 \%$ over VP9 at the same perceived quality. For future work, the compression efficiency of HEVC and VP9 with the other standardized transfer function, ARIB STD-B67, should be evaluated. Furthermore, we will study the possibility of a backward compatible solution 

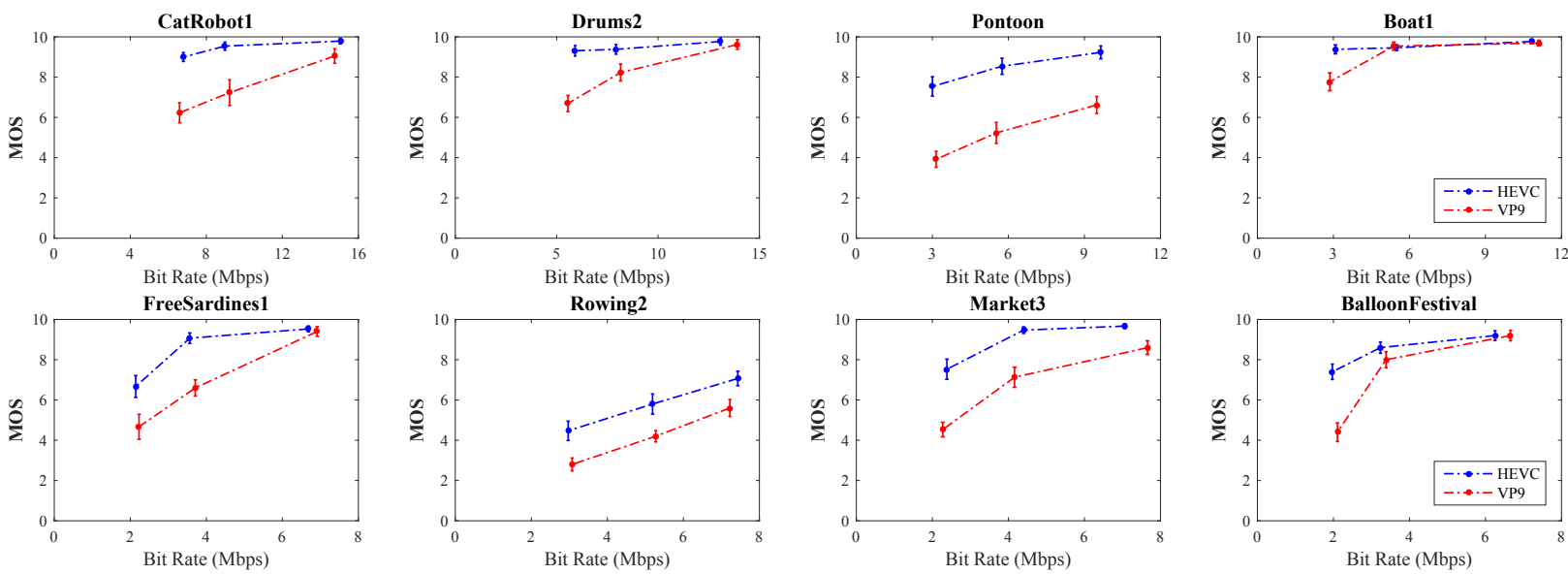

Fig. 3. Subjective evaluation results for UHD and HD content, with associated $95 \%$ confidence intervals.

including the tone expansion operator used in this paper and the HEVC video codec.

\section{ACKNOWLEDGEMENT}

This work has been conducted within the Institute of Research and Technology $\mathrm{b}<>$ com. It has been funded by the French government through the National Research Agency (ANR) investment referenced ANR-A0-AIRT-07. We would like to thank Cambodge Bist of $\mathrm{b}<>$ com for providing the upscaled HDR content. The authors are also grateful to Technicolor, Orange, Envivio, sea events, Thomson Video Networks, TDF, L'Oreille du Chat and Pôle Image et Réseaux for providing the video content used in this work.

\section{REFERENCES}

[1] J. A. Ferwerda, "Elements of Early Vision for Computer Graphics," IEEE Computer Graphics and Applications, vol. 21-5, pp. 22-33, 2001.

[2] G. J. Sullivan, J.-R. Ohm, W.-J. Han, and T. Wiegand, "Overview of the High Efficiency Video Coding (HEVC) Standard," IEEE Trans. Circuits and Systems for Video Technology, vol. 22-12, pp. 1649-1668, 2012.

[3] D. Mukherjee, J. Bankoski, A. Grange, J. Han, J. Koleszar, P. Wilkins, Y. Xu, and R. Bultje, "The Latest Open-Source Video Codec VP9 An Overview and Preliminary Results," in Picture Coding Symposium (PCS), 2013. IEEE, 2013, pp. 390-393.

[4] T. Wiegand, G. Sullivan, G. Bjontegaard, and A. Luthra, "Overview of the H.264/AVC Video Coding Standard," IEEE Trans. Circuits and Systems for Video Technology, vol. 13, no. 7, pp. 560-576, Jul. 2003.

[5] "VP8/VP9 Source Code." [Online]. Available: https://chromium. googlesource.com/webm/libvpx/

[6] S. Miller, M. Nezamabadi, and S. Daly, "Perceptual Signal Coding for More Efficient Usage of Bit Codes," SMPTE Motion Imaging Journal, vol. 122-4, pp. 52-59, 2013.

[7] T. Borer, "Non-linear Opto-Electrical Transfer Functions for High Dynamic Range Television," BBC Research and Development White Paper.

[8] SMPTE ST.2084:2014, "High Dynamic Range Electro-Optical Transfer Function of Mastering Reference Displays," 2014.

[9] ARIB STD-B67, "Essential Parameter Values for the Extended Image Dynamic Range (EIDRTV) System for Programme Production," 2015.

[10] M. Řeřábek and T. Ebrahimi, "Comparison of Compression Efficiency Between HEVC/H.265 and VP9 Based on Subjective Assessments," Proceedings of SPIE, Applications of Digital Image Processing XXXVII, vol. 9217, p. 92170U, August 2014.

[11] D. Grois, D. Marpe, A. Mulayoff, B. Itzhaky, and O. Hadar, "Performance Comparison of H.265/MPEG-HEVC, VP9, and H.264/MPEGAVC Encoders," in Picture Coding Symposium (PCS), 2013. IEEE, 2013, pp. 394-397.
[12] M. Azimi, R. Boitard, B. Oztas, S. Ploumis, H. R. Tohidypour, M. T. Pourazad, and P. Nasiopoulos, "Compression Efficiency of HDR/LDR Content," in Quality of Multimedia Experience (QoMEX), 2015 Seventh International Workshop on. IEEE, 2015, pp. 1-6.

[13] A. Luthra, E. François, and W. Husak, "Call for Evidence (CfE) for HDR and WCG Video Coding," ISO/IEC JTC1/SC29/WG11 MPEG2014/N15083, Geneva, Switzerland, February 2015.

[14] J. Strom, J. Sole, and Y. He, "Report of HDR CE1," ISO/IEC JTC1/SC29/WG11 MPEG2016/m37605, San Diego, USA 2016.

[15] T. K. Tan, R. Weerakkody, M. Mrak, N. Ramzan, V. Baroncini, J.-R. Ohm, and G. J. Sullivan, "Video Quality Evaluation Methodology and Verification Testing of HEVC Compression Performance," IEEE Trans. Circuits and Systems for Video Technology, vol. 26-1, pp. 76-90.

[16] J.-R. Ohm, G. J. Sullivan, H. Schwarz, T. K. Tan, and T. Wiegand, "Comparison of the Coding Efficiency of Video Coding StandardsIncluding High Efficiency Video Coding (HEVC)," IEEE Trans. Circuits and Systems for Video Technology, vol. 22-12, pp. 1669-1684, 2012.

[17] F. Kainz and R. Bogart, "Technical Introduction to OpenEXR," Industrial Light \& Magic, vol. 18, 2009.

[18] A. M. Tourapis, Y. Su, and D. Singer, "HDRTools: Extensions and Improvements," ISO/IEC JTC1/SC29/WG11 MPEG2016/m37681, San Diego, USA 2016.

[19] J. Samuelsson, M. Petersson, J. Strom, and K. Andersson, "Using Chroma QP Offset on HDR Sequences," ISO/IEC JTC1/SC29/WG11 MPEG2015/m36581, Warsaw, Poland, June 2015.

[20] K. Andersson, P. Hermansson, J. Samuelsson, J. Strom, and M. Petersson, "Report for CE1.a (Chroma QP)," ISO/IEC JTC1/SC29/WG11 MPEG2015/m37179, Geneva, Switzerland, October 2015.

[21] J. Zhao, S.-H. Kim, A. Segall, and K. Misra, "Performance Investigation of High Dynamic Range and Wide Color Gamut Video Coding Techniques," ISO/IEC JTC1/SC29/WG11 MPEG2015/m37439, Geneva, Switzerland, October 2015

[22] C. Bist, R. Cozot, G. Madec, and X. Ducloux, "Style Aware Tone Expansion for HDR Displays," in Proceedings of Graphics Interface 2016. Canadian Information Processing Society, 2016.

[23] "HEVC Reference Software." [Online]. Available: https://hevc.hhi. fraunhofer.de/svn/svn_HEVCSoftware/

[24] "VP8 Encoder Parameter Guide." [Online]. Available: http://www. webmproject.org/docs/encoder-parameters/

[25] M. Narwaria, M. P. Da Silva, and P. Le Callet, "HDR-VQM: An Objective Quality Measure for High Dynamic Range Video," Signal Processing: Image Communication, vol. 35, pp. 46-60, 2015.

[26] H. R. Sheikh and A. C. Bovik, "Image Information and Visual Quality," IEEE Trans. Image Processing, vol. 15-2, pp. 430-444, 2006.

[27] G. Bjøntegaard, "Calculation of Average PSNR Differences Between RD-curves," VCEG-M33 ITU-T Q6/16, Austin, TX, USA, April, 2001.

[28] "Subjective Video Quality Assessment Methods for Multimedia Applications," ITU-T Rec. P910, September 1999.

[29] "Methodology for the Subjective Assessment of the Quality of Television Pictures," ITU-R Rec. BT500, January 2012. 